\title{
Hippocampal Hyperactivation Associated with Cortical Thinning in Alzheimer's Disease Signature Regions in Non-Demented Elderly Adults
}

\author{
Deepti Putcha, ${ }^{1,2}$ Michael Brickhouse, ${ }^{2}$ Kelly 0'Keefe, ${ }^{1,2}$ Caroline Sullivan, ${ }^{1,2}$ Dorene Rentz, ${ }^{1,2}$ Gad Marshall, ${ }^{1,2}$ \\ Brad Dickerson, ${ }^{2}$ and Reisa Sperling ${ }^{1,2}$ \\ ${ }^{1}$ Center for Alzheimer Research and Treatment, Department of Neurology, Brigham and Women's Hospital, Harvard Medical School, Boston, \\ Massachusetts 02115, and 2Department of Neurology and Athinoula A. Martinos Center for Biomedical Imaging, Massachusetts General Hospital, Boston, \\ Massachusetts 02114
}

\begin{abstract}
Alzheimer's disease (AD) is associated with functional and structural alterations in a distributed network of brain regions supporting memory and other cognitive domains. Functional abnormalities are present in mild cognitive impairment (MCI) with evidence of early hyperactivity in medial temporal lobe regions, followed by failure of hippocampal activation as dementia develops. Atrophy in a consistent set of cortical regions, the "cortical signature of $\mathrm{AD}$," has been reported at the stage of dementia, MCI, and even in clinically normal $(\mathrm{CN})$ older individuals predicted to develop AD. Despite multiple lines of evidence for each of these findings, the relationship between this structural marker of AD-related neurodegeneration and this functional marker of the integrity of the episodic memory system has not yet been elucidated. We investigated this relationship in 34 nondemented older humans $(\mathrm{CN}, N=18 ; \mathrm{MCI}, N=16)$. Consistent with previous studies, we found evidence of hippocampal hyperactivation in MCI compared with CN. Additionally, within this MCI group, increased hippocampal activation correlated with cortical thinning in AD-signature regions. Even within the $\mathrm{CN}$ group, increased hippocampal activity was negatively correlated with cortical thinning in a subset of regions, including the superior parietal lobule $(r=-0.66 ; p<$ 0.01). These findings, across a continuum of nondemented and mildly impaired older adults, support the hypothesis that paradoxically increased hippocampal activity may be an early indicator of AD-related neurodegeneration in a distributed network.
\end{abstract}

\section{Introduction}

The most salient clinical feature of early Alzheimer's disease (AD) is failure of episodic memory. Recent neuroimaging studies have indicated that early $\mathrm{AD}$ is associated with both functional abnormalities of the hippocampus and cortical atrophy in a distributed memory network (Nestor et al., 2004; Ries et al., 2008). Converging evidence suggests that the neurodegenerative process begins well before clinical manifestations of AD pathology become apparent, and likely continues to evolve over the course of mild cognitive impairment (MCI) (Holland et al., 2009). The nature of the relationship between early hippocampal dysfunction and early neurodegeneration in a distributed cortical memory network remains largely unknown.

The functional magnetic resonance imaging (fMRI) studies in MCI published to date have yielded variable results. Several

Received Sept. 16, 2011; accepted 0ct. 2, 2011

Author contributions: R.S. designed research; D.P., K.O., C.S., D.R., G.M., B.D., and R.S. performed research; D.P., M.B., and B.D. analyzed data; D.P., B.D., and R.S. wrote the paper.

Conflict of Interest: Dr. Sperling has consulted for Avid (unpaid), Bayer, Bristol-Myers-Squibb, Eisai, Elan, Janssen, and Pfizer, but not in a manner directly relevant to this manuscript.

This work was supported by the National Institute on Aging Grants R01 AG-027435, P01 AG036694, K23 AG027171, and P50 AG005134. We gratefully acknowledge Mary Foley, Larry White, and the Athinoula A. Martinos Center for Biomedical Imaging for assistance with scan acquisition. We are also grateful for the participation of our research subjects.

Correspondence should be addressed to Dr. Reisa Sperling, Center for Alzheimer Research and Treatment, Brigham and Women's Hospital, 221 Longwood Avenue, Boston, MA 02115. E-mail: reisa@rics.bwh.harvard.edu. DOI:10.1523/JNEUROSCI.4740-11.2011

Copyright $\odot 2011$ the authors $\quad 0270-6474 / 11 / 3117680-09 \$ 15.00 / 0$ groups have demonstrated evidence of decreased activity, or hypoactivation, in MCI similar to that reported in AD dementia (Machulda et al., 2003; Johnson et al., 2006; Petrella et al., 2006), while other groups have reported increased activation, or hyperactivation, in MCI subjects compared with age-matched controls (Dickerson et al., 2004, 2005; Celone et al., 2006; Hämäläinen et al., 2007; Kircher et al., 2007; Yassa et al., 2010). There is also evidence of medial temporal lobe (MTL) hyperactivity in individuals at genetic or familial risk for AD (Bookheimer et al., 2000; Bondi et al., 2005; Bassett et al., 2006; Filippini et al., 2009; Quiroz et al., 2010). Our group has also observed hippocampal hyperactivity in low-performing, clinically normal $(\mathrm{CN})$ adults (Miller et al., 2008b) and amyloid-positive, mildly impaired older adults (Sperling et al., 2009). Given this controversy in the literature, the present study aimed to determine whether increased hippocampal activity is an early marker of memory dysfunction in the setting of more widespread neurodegeneration.

Several cortical regions, including the inferior temporal gyrus and areas of the medial and lateral parietal cortices, form an $\mathrm{AD}$ signature pattern of cortical thinning (Buckner et al., 2005; Du et al., 2007; Dickerson et al., 2009). Greater atrophy of these cortical regions in MCI is associated with greater likelihood of further cognitive decline toward AD dementia (Bakkour et al., 2009), and even in $\mathrm{CN}$ adults destined to develop $\mathrm{AD}$ dementia many years later (Dickerson et al., 2011). Similarly, hippocampal hyperactivity has been reported to predict cognitive decline in MCI subjects 
(Miller et al., 2008a; O'Brien et al., 2010). However, the relationship between cortical atrophy and hippocampal function across a continuum of nondemented older adults remains to be elucidated.

The present study aimed to investigate whether hippocampal hyperactivation is an indicator of early neurodegeneration in a distributed memory network thought to be vulnerable to $\mathrm{AD}$ pathology. Since the AD-signature pattern of multifocal cortical thinning is thought to be a sensitive and specific marker of $\mathrm{AD}$ neurodegeneration, we sought here to directly link hippocampal hyperactivation to the expression of this marker. Specifically, we hypothesized that across a range of nondemented older adults, greater hippocampal activation would be associated with greater thinning in $\mathrm{AD}$ signature cortical regions and with worse memory performance.

\section{Materials and Methods}

Subjects. Thirty-four nondemented right-handed older adults (16 males; mean age, 74.2 years; age range, $61-83$ years) participated in this study and provided informed consent in accordance with the Human Research Committee guidelines of Brigham and Women's Hospital and Massachusetts General Hospital. All subjects were screened for neurological and psychiatric illness. Subjects were recruited from on-going longitudinal studies of aging and from neurology clinics and were screened for contraindications to MRI. At study entry, the Mini-Mental State Examination (MMSE) was administered and every subject scored between 26 and 30. Upon entering the study, subjects were classified based on the Clinical Dementia Rating (CDR) scale (Morris, 1993) as well as the Wechsler Memory Scale Logical Memory IIa. The CDR score was based on an interview with the subject and the subject's healthy study partner with whom the subject had daily contact. Eighteen subjects were classified as "cognitively normal" with a global CDR of 0 , and 16 subjects were classified as having "mild cognitive impairment" with a global CDR of 0.5 . The subjects selected for this study were classified as CDR 0.5 based on reports of subjective memory complaints, corroborated by study partners. These individuals were classified as MCI as per the new Alzheimer's Disease Neuroimaging Initiative criteria for early and late MCI. None of the subjects met clinical criteria for dementia.

Neuroimaging procedure. Each scanning session was performed in the mid-morning hours and included $25 \mathrm{~min}$ of structural imaging sequences followed by the functional cognitive paradigm. The fMRI paradigm used was a mixed block and event-related design adapted from a previously published fMRI block design paradigm (Celone et al., 2006), and a subsequent memory event-related design (Miller et al., 2008b). Subjects were scanned during encoding runs consisting of alternating blocks of novel and repeated face-name pairs, interspersed with visual fixation on a white crosshair. There were six runs in total. In each run, there were two novel blocks with seven novel face-name pairs each and two repeated blocks with two alternating repeated face-name pairs (one male and one female) each, resulting in a total of 84 presentations of novel face-name pairs and 84 presentations of the repeated face-name pairs (42 repetitions of each). The stimulus duration of the novel and repeated faces within each block were jittered, with optimal timings derived from OptSeq (Dale, 1999). Faces were each displayed for $4.5 \mathrm{~s}$ against a black background with a fictional first name printed in white letters underneath. During the presentation of each face-name pair, subjects were asked to press a button indicating whether they thought the name was a "good" name for the face or not, a purely subjective task designed to enhance associative encoding (Sperling et al., 2003). Before each run, subjects were explicitly instructed to try to remember the name that was associated with each face. Face-name stimuli were randomly intermixed with trails of visual fixation varying in duration from 0.3 to $2.2 \mathrm{~s}$. Between each block of novel and repeated face-name pairs, visual fixation was presented for $25 \mathrm{~s}$. Visual stimuli were presented using MacStim 2.5 software (WhiteAnt Occasional Publishing). Images were projected through a collimating lens onto a mirrored screen attached to the head coil. Task responses were collected using a fiber-optic response box held in the right hand. Cushions were in place to help minimize subject movement, and headphones were used to communicate with the subjects during the scan and to help dampen scanner noise.

Postscan memory testing. After the scanning session, subjects underwent a forced-choice recognition memory test in which the 84 novel faces and two repeated faces seen during the scan session were presented on a computer screen. Each face was paired with two names underneath in a counter-balanced design: one name was correctly paired with the face and the other had been paired with a different face during the scan session. Subjects were asked to indicate which of the two names was correctly paired with each face and to indicate whether they felt high or low confidence about their answer.

Image acquisition. Subjects were scanned using a Siemens Trio 3T scanner (Siemens Medical Systems). $\mathrm{T}_{1}$-weighted structural images were acquired using a magnetization prepared-rapid acquisition gradient echo (MP-RAGE) sequence [repetition time (TR), $2300 \mathrm{~ms}$; echo time (TE), $2.98 \mathrm{~ms}$; inversion time, $900 \mathrm{~ms}$; flip angle (FA), $9^{\circ}$; field of view (FOV), $256 \mathrm{~mm}$; matrix, $240 \times 256$; slice thickness, $1.20 \mathrm{~mm}$; 160 sagittal slices (right to left)]. In addition, blood oxygen level-dependent (BOLD) fMRI data were acquired using a $\mathrm{T}_{2}{ }^{*}$-weighted gradient-echo echoplanar imaging (EPI) sequence [TR, $2000 \mathrm{~ms}$; TE, $30 \mathrm{~ms}$; FA, 90\% FOV, $200 \mathrm{~mm}$; matrix, $64 \times 64$ (in-plane resolution $3.1 \times 3.1 \mathrm{~mm}^{2}$ )]. Thirty oblique coronal (anterior to posterior) slices with a thickness of $5.0 \mathrm{~mm}$ and an interslice gap of $1.0 \mathrm{~mm}$ were acquired and oriented perpendicular to the anterior commissural-posterior commissural (AC-PC) line. A total of six functional runs per scan session were acquired, each consisting of 127 whole-brain acquisitions with five TR discarded for $\mathrm{T}_{1}$ stabilization. The total functional scanning time was $25.44 \mathrm{~min}$, with the acquisition time of each run being 4.24 min.

Image processing and analysis. MP-RAGE images were processed through the FreeSurfer (version 4.5.0) pipeline (http://surfer.nmr.mgh. harvard.edu). As part of this semiautomated pipeline, preprocessing of structural volumes included an affine registration to Talairach space, bias field correction, and removal of skull and dura surrounding the brain. Each volume underwent minimal manual editing (e.g., manual deletion of strips of skull that were erroneously included in the pial surface) to ensure that the white matter and pial surfaces were properly reconstructed. A single individual, blinded to the diagnosis, completed these edits. A second individual completed a visual inspection of the edits to ensure proper segmentation but no additional edits were made. All other processing steps followed the default parameters and were fully automated. fMRI data were preprocessed using Statistical Parametric Mapping (SPM2; Wellcome Department of Cognitive Neurology, London, UK) for MatLab (Mathworks). Functional data were reoriented to the AC-PC line, then realigned using INRIAlign, a motion-correction algorithm unbiased by local signal changes. The data were then normalized to the standard SPM2 EPI template and resampled into a $3 \mathrm{~mm}$ isotropic resolution in MNI305 space. Data were smoothed using a $8 \mathrm{~mm}$ Gaussian kernel. No scaling was implemented for global effects. A high-pass filter of $260 \mathrm{~s}$ was used to remove low-frequency signal (e.g., drifts across an entire fMRI run). The data were then modeled by convolving a canonical hemodynamic response function with the onsets from encoded facename pairs.

Trials were analyzed in an event-related design format and were categorized based on responses on the postscan memory test. A trial was classified as a high-confidence hit $(\mathrm{HCH})$ if the subject chose the correct name and reported high confidence, a high-confidence miss if the subject chose the incorrect name but reported high confidence, a low-confidence hit if the subject chose the correct name but reported low confidence, or a low-confidence miss if the subject chose the incorrect name and reported low confidence. The activation contrast of interest was defined as $\mathrm{HCH}$ versus a fixation cross $(\mathrm{HCHvF})$. These event-related analyses were based on a mixed-effects general linear model in SPM2. We chose this contrast because of our own previous work and that of others documenting robust hippocampal activation during successful encoding compared with a fixation cross (Kircher et al., 2007; Miller et al., 2008b). We did not use a Hits-versus-Misses contrast to define hippocampal activation for this study because this mixed block and event-related design did not yield robust hippocampal activation for this contrast at either time point, even 


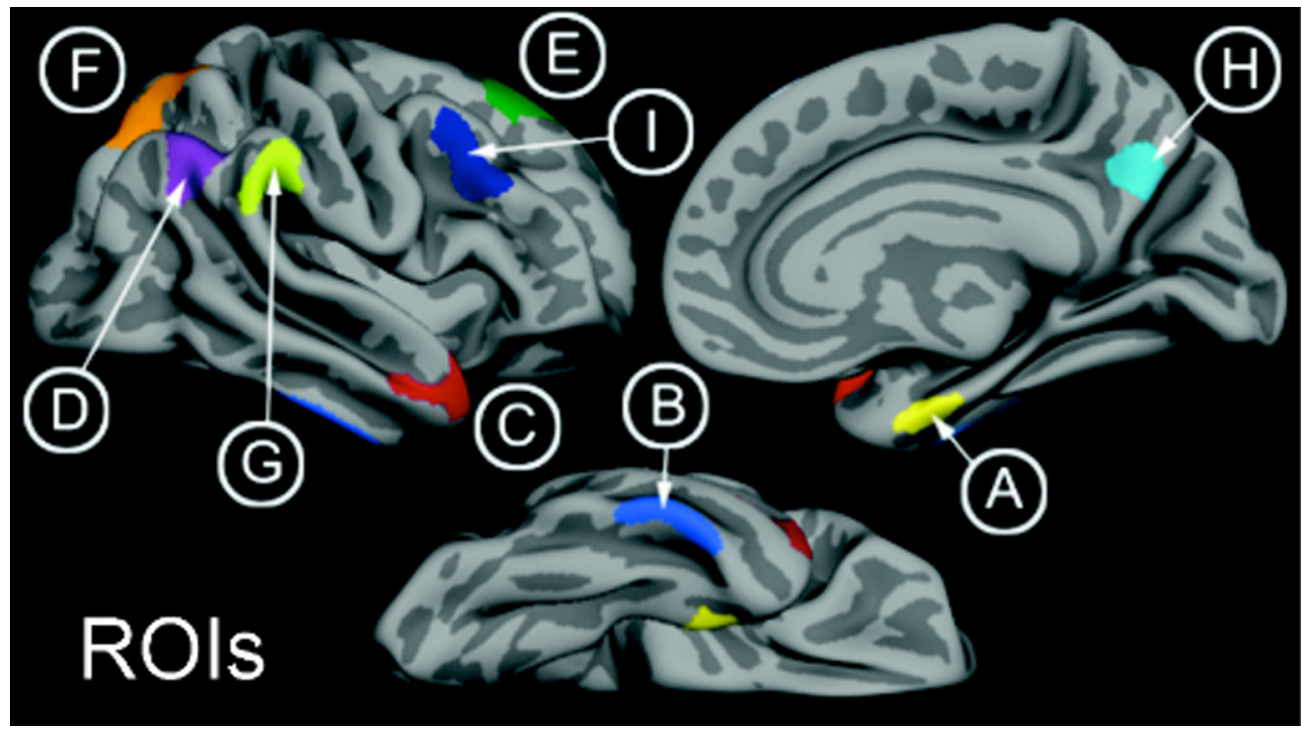

Figure 1. AD signature of cortical thinning. A, Medial temporal lobe; $B$, inferior temporal gyrus; $C$, temporal pole; $D$, angular gyrus; $E$, superior frontal gyrus; $F$, superior parietal lobule; $G$, supramarginal gyrus; $H$, precuneus; $I$, inferior frontal sulcus.

with young subjects. This is likely due to the limited jitter possible within the block timing constraints, as well as the relatively small number of stimuli in the miss trials in normal subjects.

Functional and structural measures. Both functional activation measures and structural measures of cortical thickness were defined within the individual's own neuroanatomy. Structural MRI data (MP-RAGE) were processed through FreeSurfer and were used to generate individual hippocampus regions of interest (ROIs) that were anatomically specific for each subject. Each subject's fMRI data were then coregistered to that subject's structural MRI data. Magnitude of activation, defined as percentage signal change (PSC), was then extracted from each individual's anatomically defined bilateral hippocampi. The hippocampus ROIs were furthermore functionally defined, as we extracted PSC only from those voxels within each individual's hippocampus that were activating at a threshold of $p<0.05$, with an extent threshold of five contiguous voxels.

FreeSurfer software was also used to delineate the thickness of the gray matter cortical ribbon (Fischl et al., 2001). This was accomplished by deriving one surface between the gray and white matter and a second surface between the gray matter and CSF. The cortical thickness for a given region was the average of distances between the two surfaces (in millimeters). An Alzheimer's disease set of regions was investigated based on prior studies in independent samples indicating where there is consistent cortical thinning in $\mathrm{AD}$ dementia patients compared with controls (Bakkour et al., 2009; Dickerson et al., 2009). We obtained cortical thickness measures from nine ROIs, including the medial temporal lobe, angular gyrus, inferior temporal gyrus, superior parietal lobule, the precuneus cortex, the temporal pole, the superior frontal gyrus, supramarginal gyrus, and middle frontal gyrus (Fig. 1). Hemisphere-averaged regions of interest were calculated for analysis. Additionally, the $\mathrm{AD}$ signature of cortical thinning was calculated as the overall raw (nonweighted) average of all nine ROIs. Details of this procedure have been described in prior publications (Bakkour et al., 2009; Dickerson et al., 2009).

We sought to test whether signal measured from the hippocampus during successful face-name pair encoding was related to cortical thickness measurements from areas that are susceptible to atrophy early in the progression of AD. All measures of cortical thickness, hippocampal volume, and hippocampal activation were extracted from hemisphereaveraged ROIs, as the values for left and right hemisphere were highly correlated with each other. We used the memory performance measure of percentage correct with high confidence $(\% \mathrm{HCH})$ instead of percentage correct to be consistent with our contrast of $\mathrm{HCHvF}$, which defined hippocampal activation. The behavioral performance measures of $\% \mathrm{HCH}$ and percentage total correct were highly correlated with each
Table 1. Subject demographics

\begin{tabular}{llll}
\hline & All & CDR O & CDR 0.5 \\
\hline$N$ & 34 & 18 & 16 \\
Male/Female & $16 / 18$ & $4 / 14$ & $12 / 4$ \\
Age (years) & $74.2 \pm 7.1$ & $73.5 \pm 6.0$ & $75.0 \pm 8.2$ \\
CDR SOB & - & $0 \pm 0$ & $1.6 \pm 1.09^{* *}$ \\
Education (years) & $17.5 \pm 2.9$ & $17.4 \pm 3.0$ & $17.6 \pm 2.8$ \\
MMSE & $29.0 \pm 1.0$ & $29.4 \pm 0.8$ & $28.4 \pm 1.1^{*}$ \\
RAVLT & $8.2 \pm 4.6$ & $11.0 \pm 3.6$ & $4.9 \pm 3.6^{* *}$
\end{tabular}

Values presented in the table are means \pm SD. RAVLT, Rey auditory verbal learning task; SOB, Sum of Boxes. ${ }^{*} p<0.05,{ }^{* *} p<0.005$.

other. First, two-sample $t$ tests were conducted to assess whether there was a group difference between the CN (CDR 0) and MCI (CDR 0.5) groups in measures of cortical thickness, hippocampal volume, and hippocampal activation, or memory performance. Next, we used Pearson correlation analyses to examine relationships between behavioral performance on the fMRI task $(\% \mathrm{HCH})$, and a summary measure of cortical thickness from the $\mathrm{AD}$ signature regions to determine whether there was a relationship between behavioral performance and cortical thickness in an $\mathrm{AD}$ phenotypic pattern. We also correlated performance with a measure of hippocampal activation $(\mathrm{HCHvF})$ to determine whether dysfunctional hippocampal activation was associated with better or worse memory performance. We then used linear regressions to investigate the relationship between hippocampal activation and cortical thickness measures, controlling for age. These regressions were examined in the whole group as well as within the CDR subgroups.

\section{Results}

Subjects

The MCI subjects exhibited a range of mild functional impairment, with CDR sum-of-boxes scores ranging from 0.5 to 4.0. The average MMSE score in the MCI group was slightly lower (28.4 of 30) than that of the CN group $(29.4 ; t=2.88, p=0.007)$. The average Rey auditory verbal learning task 30 min delayed recall memory score over the whole sample was 8.2 (of 15.0), with the average of the MCI group being lower (4.9) than the $\mathrm{CN}$ group $(11.0 ; t=5.19, p<0.001)$. Although there were differences in the gender ratio between the $\mathrm{CN}$ and MCI groups, there were no statistically significant gender differences in any variable of interest. Demographic data are presented in Table 1. 
Table 2. Behavioral performance

\begin{tabular}{llll}
\hline & \multicolumn{2}{l}{ Percentage of trials (SE) in each bin } \\
\cline { 2 - 4 } & All & CDR 0 & CDR 0.5 \\
\hline Hits, high confidence & $34.4(3.2)$ & $43.1(3.5)$ & $25.6(4.5)$ \\
Hits, low confidence & $28.6(2.2)$ & $24.4(2.5)$ & $33.4(3.4)$ \\
Miss, high confidence & $12.3(1.5)$ & $13.7(1.8)$ & $11.8(2.4)$ \\
Miss, low confidence & $22.6(2.05)$ & $18.2(2.0)$ & $27.6(3.4)$ \\
\hline
\end{tabular}

Values presented are means (SEs) for each trial.

a

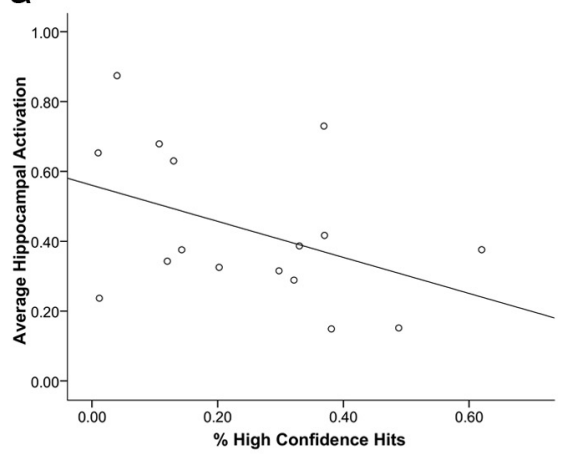

C

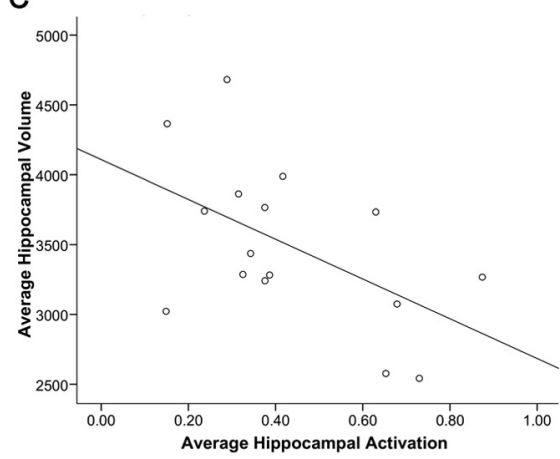

b

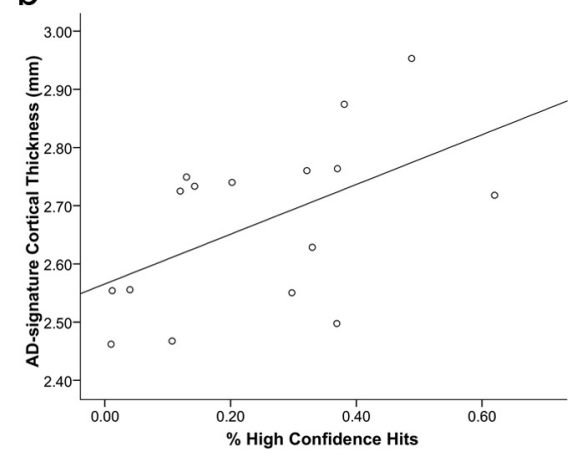

Figure 2. In MCl, memory test performance (percentage high confidence hits) is (a) negatively related to hippocampal activation $(r=-0.43, p=0.09)$ and $(\boldsymbol{b})$ positively related to AD signature cortical thickness $(r=0.38, p=0.03)$. Additionally, (c), Hippocampal activation is negatively related to hippocampal volume $(r=-0.53, p=0.04)$.

\section{fMRI task memory test performance}

A summary of performance on the postscan forced-choice recognition memory test is presented in Table 2. The MCI group $(59.0 \%$ correct) performed worse than the $\mathrm{CN}$ group $(67.5 \%$ correct) on successfully remembering face-name pairs $(t=2.77$, $p=0.009)$ and on successfully remembering face-name pairs with high confidence $(t=3.27, p=0.003)$. We first analyzed memory performance (percentage high confidence hits) on the postscan memory task in relation to hippocampal fMRI activation. In the full sample of subjects, there was a negative relationship such that greater hippocampal activity was paradoxically related to worse task performance $(r=-0.341, p=0.048)$. Importantly, this relationship was not present in the CN group ( $r=$ $0.12, p>0.5)$, but was present at a trend level in the MCI group $(r=-0.43, p=0.09$; Fig. 2). Furthermore, we conducted separate linear regression analyses to determine whether memory performance predicted hippocampal activation when hippocampal volume was controlled for. When raw hippocampal volume was entered into the regression model, memory performance was still related to hippocampal activation $(\beta=-0.335, p=0.048)$. However, if intracranial volume (ICV) was controlled for in the measure of hippocampal volume, then memory performance no longer predicted hippocampal activation $(\beta=-0.229, p=0.185)$, reflecting that ICV-adjusted hippocampal volume is colinear with memory performance $(r=0.34, p=0.05)$.

We next analyzed memory performance on the postscan memory test in relation to a summary $\mathrm{AD}$ signature cortical thickness measure. In the full sample, AD signature cortical thickness was directly correlated with memory performance during the postscan task for percentage high confidence hits $(r=$ 0.38, $p=0.03)$. Again, this effect was driven by the MCI group such that there was no relationship with the memory performance variable in the $\mathrm{CN}$ group ( $r=$ $0.25, p>0.3)$ and a strong relationship in the MCI group ( $r=0.53, p=0.03$; Fig. 2 ).

\section{Hippocampal hyperactivation is associated with MTL atrophy in MCI} The MCI group showed hippocampal hyperactivation compared with the $\mathrm{CN}$ group ( $t=2.05, p=0.04$; Fig. 3 ). The two groups did not differ in hippocampal volume. The average hippocampal volume for the $\mathrm{CN}$ group was $3405.33 \pm 88.2$ (mean \pm SE, in cubic millimeters) and the MCI group was $3491.62 \pm 145.7$, which was not significantly different $(t=$ $-0.520, p>0.5)$. Likewise, there was no statistical difference between left and right hippocampal volumes either within or between groups $(p>0.5)$. Furthermore, when ICV was accounted for, the $\mathrm{CN}$ and MCI groups still showed no differences in hippocampal volume $(t=1.08, p>0.2)$. Within the MCI group alone, however, there was a strong inverse relationship between hippocampal volume and activation ( $r=-0.53, p=0.04$; Fig. 2$)$, such that, paradoxically, a smaller hippocampus was related to greater hippocampal activation. There was no relationship between hippocampal activation and hippocampal volume in the combined sample or in the $\mathrm{CN}$ group alone (all $p>0.1$ ). When correcting hippocampal volumes for $\mathrm{ICV}$, however, this effect is made more dramatic. Within the MCI group alone, there is an even stronger inverse relationship between hippocampal volume and activation $(r=-0.71, p=$ $0.002)$, which drives this relationship to be significant in the overall sample $(r=-0.41, p=0.015)$, yet within the $\mathrm{CN}$ group alone, this relationship is still nonsignificant $(r=0.12, p>0.5)$.

Analyses of the cortical thickness data indicated that this relatively early MCI group exhibited very early neurodegenerative change consistent with $\mathrm{AD}$, particularly in the medial temporal lobe where there was an indication of thinning compared with the CN group ( $p>0.1$; Fig. 3$)$. With the exception of the precuneus cortex $(t=2.11, p=0.04)$, where the MCI group (mean, $\sim 2.58 \mathrm{~mm}$ ) had thicker cortex than the $\mathrm{CN}$ group (mean, $\sim 2.47 \mathrm{~mm}$ ), there were no other significant group differences in thickness.

Hippocampal hyperactivation is associated with $\mathrm{AD}$ signature cortical atrophy

Over the full continuum of nondemented older adults, increased hippocampal activity was significantly related to reduced cortical 
a

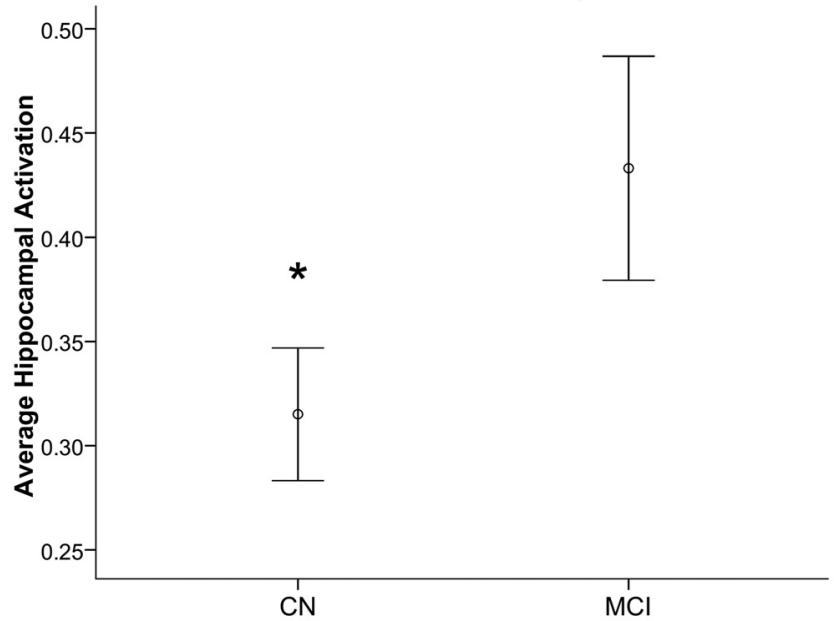

b

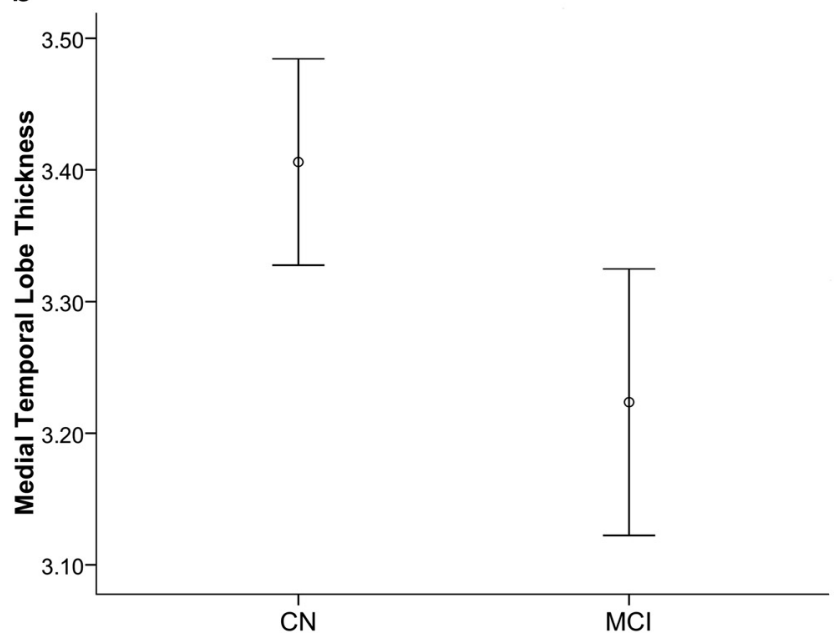

Figure 3. $\quad \mathrm{CN}(\mathrm{CDR} 0)$ versus $\mathrm{MCI}(\mathrm{CDR} 0.5)$ describing (a) hippocampal activation percentage signal change $(t=2.05, p=0.04)$ and $(\boldsymbol{b})$ medial temporal lobe cortical thickness in millimeters $(\boldsymbol{b}) .{ }^{*}$ indicates a significant difference between the means.

thickness in the summary AD signature ROI measure $(r=-0.54$, $p=0.001$; Fig. 4). Within the component ROIs that make up the $\mathrm{AD}$ signature, we observed an inverse relationship between hippocampal signal change and cortical thickness in the medial temporal lobe $(r=-0.42, p=0.01)$, as well as in multiple other cortical regions (Table 3 ). There was also a trend level association in the precuneus cortex $(r=-0.30, p=0.08)$. Controlling for age did not change these relationships significantly, suggesting that age does not mediate the relationship between hippocampal activity and cortical thinning. Exploratory whole-brain analyses confirmed that greater hippocampal activation was correlated with thinner cortex in several regions that are part of the distributed memory network within the cortical signature of $\mathrm{AD}$ (Fig. 5).

Interestingly, we observed different patterns of cortical thinning related to hippocampal activation across the two clinical subgroups. Supporting the hypothesis of this study, we observed an inverse relationship between hippocampal activity and cortical thickness in the summary $\mathrm{AD}$ signature measure within the MCI group specifically $(r=-0.67, p=0.004$; Table 3$)$; the $\mathrm{CN}$ group did not show this relationship $(p>0.1)$. That is, MCI patients, in whom we observed a greater degree of structural atrophy suggestive of $\mathrm{AD}$-related neurodegeneration, also dem- a

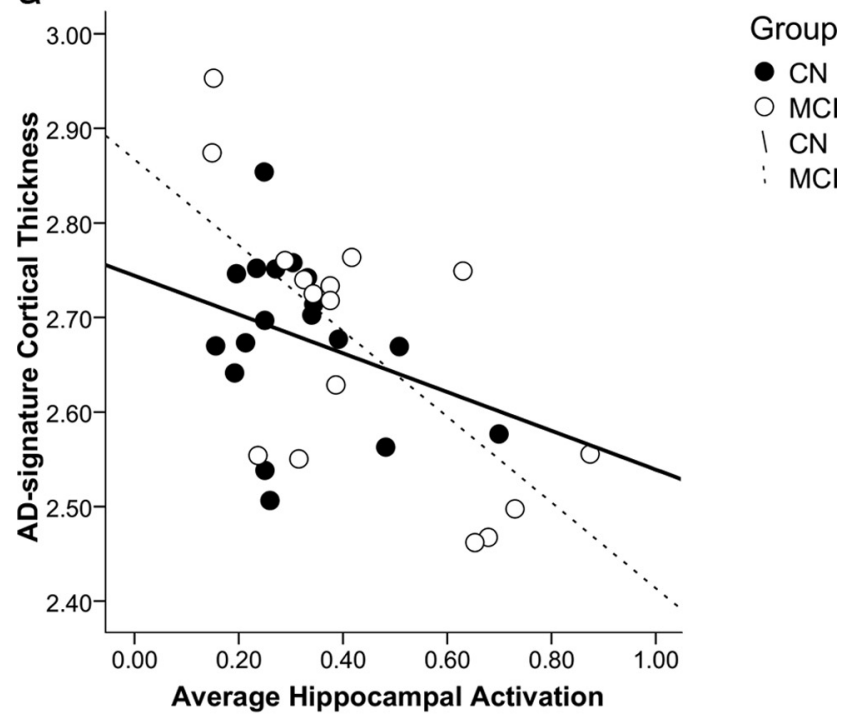

b

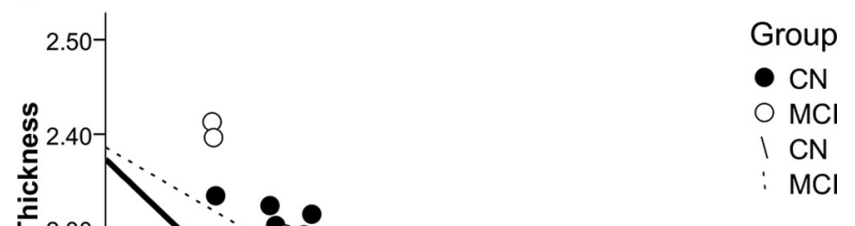
centage signal change) and cortical thickness in the (a) AD signature region summary measure (in millimeters) and (b) superior parietal lobule cortical thickness (in millimeters).

Table 3. Correlation values (Pearson's $R$ ) of average task-related hippocampal activation correlated with each structural region of interest

\begin{tabular}{llll}
\hline ROIs & All $(N=34)$ & CDR O $(N=18)$ & CDR 0.5 $(N=16)$ \\
\hline AD summary region & $-0.544^{* *}$ & -0.313 & $-0.671^{* *}$ \\
Medial temporal lobe & $-0.422^{*}$ & -0.342 & -0.397 \\
Angular gyrus & $-0.427^{*}$ & $-0.465^{*}$ & $-0.509^{*}$ \\
Inferior temporal gyrus & $-0.468^{* *}$ & -0.446 & $-0.561^{*}$ \\
Superior parietal lobule & $-0.502^{* *}$ & $-0.657^{* *}$ & $-0.625^{*}$ \\
Precuneus & -0.302 & -0.298 & $-0.552^{*}$ \\
Temporal pole & -0.005 & $0.516^{*}$ & -0.139 \\
Superior frontal gyrus & -0.214 & 0.184 & $-0.660^{* *}$ \\
Supramarginal gyrus & $-0.415^{*}$ & -0.227 & $-0.519^{*}$ \\
Middle frontal gyrus & -0.154 & 0.142 & $-0.532^{*}$ \\
\hline
\end{tabular}

${ }^{*} p<0.05,{ }^{* *} p<0.005$.

onstrated increased hippocampal activation during successful memory encoding. In examining this relationship within the specific ROIs in MCI, we found similar inverse correlations between hippocampal activation and cortical thickness of the superior 


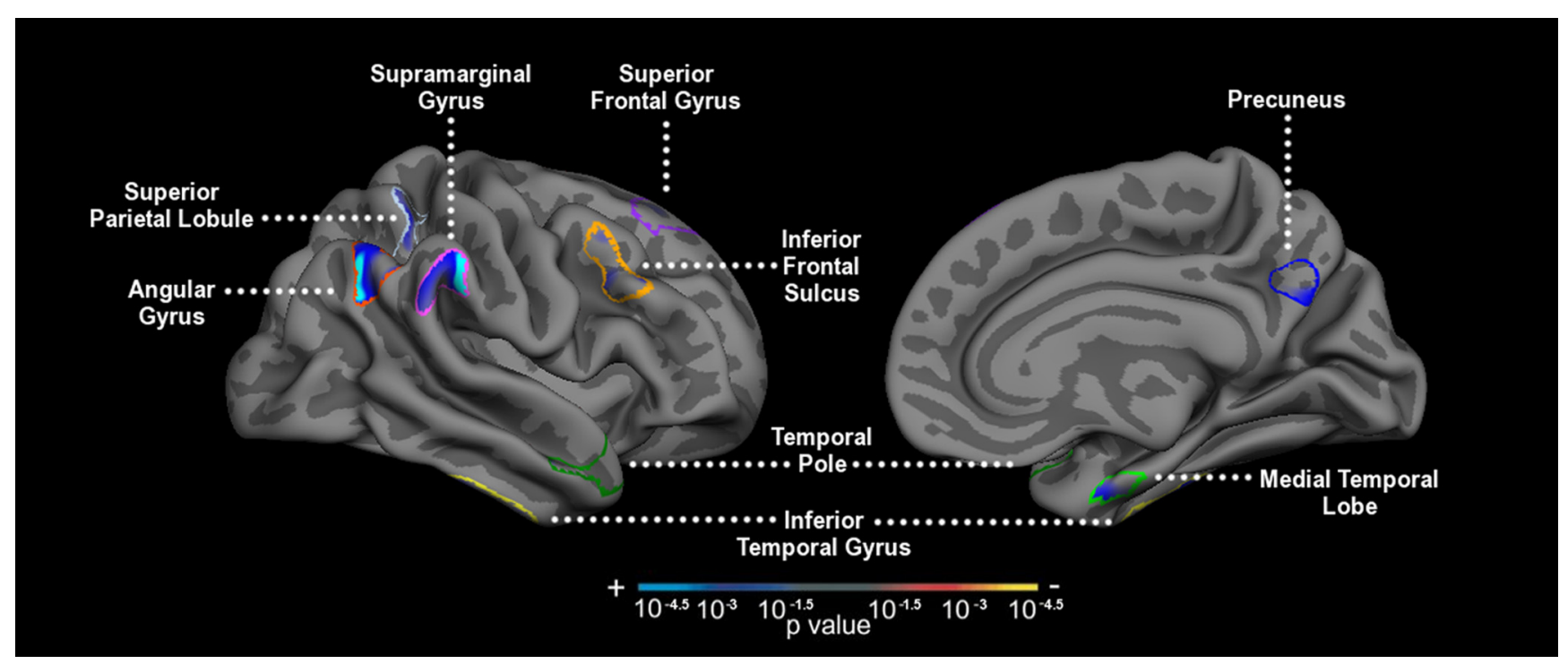

Figure 5. ROI-restricted vertex-based thickness correlation map of hippocampal activation and cortical thickness ( $p<0.01$, uncorrected) with an AD signature mask applied in the whole sample.

frontal gyrus $(r=-0.66, p=0.005)$, inferior temporal gyrus $(r=$ $-0.56, p=0.024)$, and precuneus $(r=-0.552, p=0.027)$, among other regions. There was also an indication of a relationship in the same direction with medial temporal lobe cortical thickness, though it was not significant $(r=-0.34, p>0.1)$. The $\mathrm{CN}$ group demonstrated a significant relationship between hippocampal activation and cortical thinning in only a subset of the regions observed in MCI. In particular, a strong inverse relationship was observed between thinning in the superior parietal lobule cortical region and hippocampal activation $(r=-0.657, p=0.003$; Fig. 4 ). While the MCI group showed a negative relationship between hippocampal activation and cortical thickness in every ROI examined, the $\mathrm{CN}$ group demonstrated a positive relationship between cortical thickness in the temporal pole and hippocampal activation $(r=0.52, p=0.03)$.

Last, to estimate effect sizes for both functional and structural measures, we calculated Cohen's $d$ for our measure of hippocampal activation as well as for all measures of cortical thickness. Our calculations revealed that effect size for hippocampal activation was 0.65 , with variable estimates for cortical thickness ranging from superior parietal lobule (0.46) to medial temporal lobe (0.52) to temporal pole $(0.54)$ to precuneus thinning $(0.73)$, which demonstrated the largest effect size.

\section{Discussion}

Based on human neuroimaging data (Dickerson et al., 2009; Seeley et al., 2009; Sperling et al., 2009) and studies with transgenic mouse models (Palop et al., 2007; Palop and Mucke, 2010), a memory network hypothesis has been articulated to elucidate the pattern of neurodegeneration in AD. This hypothesis posits that as large-scale memory circuits begin to undergo neurodegenerative change early in the course of $\mathrm{AD}$, both functional and structural abnormalities occur that disrupt episodic memory processes. The present investigation directly tested this hypothesis in a group of nondemented older individuals across a range of memory impairment. We demonstrated that within this group, more prominent expression of the signature marker of $\mathrm{AD}$-related neurodegenerative structural change in the cerebral cortex is related to overactivation of the hippocampus.

Confirming our previous studies with non-overlapping groups of subjects (Dickerson et al., 2005; Celone et al., 2006;
O'Brien et al., 2010), we found that early MCI individuals demonstrated increased hippocampal activity specifically during successful memory encoding compared with $\mathrm{CN}$ older adults. Although the literature in young subjects suggests that increased MTL activity is generally related to better performance on memory tasks (Brewer et al., 1998; Morcom et al., 2003), in this mixed sample of older $\mathrm{CN}$ and MCI individuals, we observed an overall negative relationship between hippocampal activation and performance on the memory task.

Recent laboratory evidence suggests that at intermediate levels of $A \beta$ pathology, presynaptic facilitation leads to synaptic potentiation and excitation, whereas at higher levels of $A \beta$, postsynaptic depression is observed (Palop and Mucke, 2010). This animal work is consistent with our current findings in humans and previous reports suggesting that the hippocampus hyperactivates early in MCI, but then demonstrates diminished signal response during episodic memory tasks in late MCI and AD dementia when considerable memory deficits are present (Celone et al., 2006). Additionally, in mouse models of AD, hyperactive neurons were found specifically in the vicinity of $A \beta$ plaques, the presence of which correlated with a decreased ability to learn successfully (Busche et al., 2008). We have previously speculated that hyperactivity could be due to a decrease in synaptic inhibition, potentially modifying the functional properties of the neurons themselves, rendering them hyperactive (Sperling et al., 2009). More recently, high-resolution fMRI has demonstrated that this hyperactivity occurs in the CA3 and dentate gyrus subfields of the hippocampus and is reflective of inefficient encoding processes (Yassa et al., 2010). Although our early work suggested that hippocampal hyperactivity might represent a compensatory response (Dickerson et al., 2004), more recent data suggest that hippocampal hyperactivity might represent evidence of neuronal excitotoxicity, and are consistent with our work suggesting that this hyperactivity may represent impending synaptic failure and incipient cognitive decline (Miller et al., 2008a; O'Brien et al., 2010). Nevertheless, it is also possible that the observed hyperactivity reflects evidence of attempted compensatory responses, a framework suggested by other studies of successful episodic memory in the aging population (Rossi et al., 2004; Duverne et al., 2009; de Chastelaine et al., 2011). 
Hippocampal hyperactivity showed a significant association with thinner cortex, specifically in the posteromedial and lateral temporoparietal cortices. This cortical pattern of thinning has been observed in AD dementia (Dickerson et al., 2009), prodromal AD (Singh et al., 2006), and in presymptomatic amyloidpositive individuals (Fjell et al., 2010; Becker et al., 2011; Sabuncu et al., 2011). There is evidence from functional and anatomical connectivity work linking the MTL to these regions of the neocortex (Kobayashi and Amaral, 2003; Vincent et al., 2006; Kahn et al., 2008; Greicius et al., 2009). Furthermore, this specific network has been implicated in playing a crucial role in episodic memory formation (Buckner et al., 2005; Sperling et al., 2010) and has been implicated as being targeted by the neurodegenerative process of AD (Seeley et al., 2009). Our findings suggest that aberrant hippocampal activity in the MTL is linked to signs of overall disrupted network health, supporting the notion of a large-scale pattern of neurodegeneration reflective of $\mathrm{AD}$ pathology. There is evidence that amyloid deposition is only moderately elevated in the MTL of AD patients (Klunk et al., 2004), and that tau-based tangle pathology is more strongly associated with hippocampal atrophy than with amyloid levels (Csernansky et al., 2004). This study adds to the literature by suggesting that these pathologies co-occur as part of a large distributed network.

Interestingly, the $\mathrm{CN}$ group specifically showed an inverse relationship between hippocampal activation and cortical thickness in lateral parietal regions. This result is consistent with findings that network dysfunction is first observed in parietal cortical regions before it is detected in temporal lobe aspects of the memory system (Desikan et al., 2010; Mormino et al., 2011). Furthermore, we noted a positive relationship between hippocampal activation and temporal pole thickness in these $\mathrm{CN}$ individuals, suggestive of "brain reserve," which may allow maintenance of memory performance. A similar pattern of enlarged temporopolar cortex was recently reported in cognitively normal elderly individuals with high amyloid burden (Chételat et al., 2010).

Converging evidence suggests that amyloid deposition in the neocortex is associated with widespread network dysfunction in preclinical and prodromal stages of AD. Drzezga et al. (2011) recently found disruptions of functional whole-brain connectivity in cortical hub regions, such as the posterior cingulate, precuneus, and temporoparietal regions, in amyloid-positive MCI subjects. At an even earlier stage, decreases in functional connectivity between these cortical areas, as well as the angular gyrus and ventral medial prefrontal cortex, have been observed in $\mathrm{CN}$ individuals with high levels of amyloid deposition (Hedden et al., 2009; Mormino et al., 2011). Our previous work with a similar face-name task in amyloid-positive individuals demonstrated hyperactivity in the default network, manifesting as failure to deactivate appropriately during encoding (Sperling et al., 2009; Vannini et al., 2011). Similarly, amyloid-positive CN have demonstrated evidence of cortical thinning primarily in neocortical regions that are key hubs in the default network (Fjell et al., 2010; Becker et al., 2011; Sabuncu et al., 2011). Consistent with the pattern of thinning in preclinical $\mathrm{AD}$, our current results suggest that hippocampal hyperactivity is associated with parietal thinning in CN. Thus, it is possible that dysfunction and neurodegeneration are more evident in the neocortices while individuals are still cognitively normal, and that MTL involvement is concurrent with the emergence of memory impairment. One recent paper from our group, however, did find evidence of MTL thinning, as well as thinning of other $\mathrm{AD}$ signature regions, in $\mathrm{CN}$ individuals who were destined to progress to dementia within a few years (Dickerson et al., 2011), suggesting that CN individuals with
MTL thinning are more likely to demonstrate imminent memory failure. Ongoing multimodal longitudinal imaging studies should serve to elucidate the temporal ordering of regionally specific neurodegeneration in the stage of preclinical AD (Sperling et al., 2011).

Despite observing hippocampal hyperactivity in the MCI group compared with the $\mathrm{CN}$ group, we did not observe significant hippocampal volume loss in the MCI group, perhaps because our MCI group is still in a relatively early stage of disease progression. Several other studies suggest that cortical thinning of the MTL structures is observed before hippocampal volume loss is evident (Bobinski et al., 1999; Dickerson et al., 2001; Killiany et al., 2002; Bakkour et al., 2009). We are actively pursuing longitudinal studies designed to determine whether both cortical thinning and hippocampal hyperactivation precede and presage neuronal loss in the hippocampus.

It is important to consider some limitations to our study. We are relying on BOLD signal as an indirect measure of hippocampal activity that may reflect other physiological properties of the aging brain, such as changes in cerebral blood volume and flow, oxygen consumption, and baseline perfusion (Logothetis and Wandell, 2004). As our sample size is relatively small, many of our tests would not survive a correction for multiple comparisons, highlighting the importance of restricting the number of ROIs examined. We chose to use AD signature ROIs in lieu of standard anatomically defined ROIs to probe regions that are particularly vulnerable to early neurodegeneration. As this was a preliminary exploration, we will continue to work toward replicating these findings in a larger sample. Lastly, longitudinal follow-up is necessary to track our participants into AD dementia to determine whether further neurodegeneration occurs in these distributed cortical regions.

This study provides evidence that hippocampal hyperactivity may be a sensitive indicator of early AD-related neurodegeneration in a functionally connected distributed cortical network known to demonstrate cortical thinning in $\mathrm{AD}$ dementia. Nondemented individuals who demonstrate hippocampal hyperactivity, one marker of impending memory decline, also show evidence of another phenotypic marker of cognitive decline, a signature of cortical thinning representative of neurodegeneration specific to $\mathrm{AD}$. Although volumetric measurements may be more feasible for large-scale use as a biomarker, this study begins to elucidate the relationship between early functional alterations in the memory system and network degeneration. Longitudinal studies are ongoing to determine whether hippocampal hyperactivity is a harbinger of future widespread network deterioration and progression to $\mathrm{AD}$ dementia.

\section{References}

Bakkour A, Morris JC, Dickerson BC (2009) The cortical signature of prodromal AD: regional thinning predicts mild AD dementia. Neurology 72:1048-1055.

Bassett SS, Yousem DM, Cristinzio C, Kusevic I, Yassa MA, Caffo BS, Zeger SL (2006) Familial risk for Alzheimer's disease alters fMRI activation patterns. Brain 129:1229-1239.

Becker JA, Hedden T, Carmasin J, Maye J, Rentz DM, Putcha D, Fischl B, Greve DN, Marshall GA, Salloway S, Marks D, Buckner RL, Sperling RA, Johnson KA (2011) Amyloid-beta associated cortical thinning in clinically normal elderly. Ann Neurol 69:1032-1042.

Bobinski M, de Leon MJ, Convit A, De Santi S, Wegiel J, Tarshish CY, Saint Louis LA, Wisniewski HM (1999) MRI of entorhinal cortex in mild Alzheimer's disease. Lancet 353:38-40.

Bondi MW, Houston WS, Eyler LT, Brown GG (2005) fMRI evidence of compensatory mechanisms in older adults at genetic risk for Alzheimer disease. Neurology 64:501-508. 
Bookheimer SY, Strojwas MH, Cohen MS, Saunders AM, Pericak-Vance MA, Mazziotta JC, Small GW (2000) Patterns of brain activation in people at risk for Alzheimer's disease. N Engl J Med 343:450-456.

Brewer JB, Zhao Z, Desmond JE, Glover GH, Gabrieli JD (1998) Making memories: brain activity that predicts how well visual experience will be remembered. Science 281:1185-1187.

Buckner RL, Snyder AZ, Shannon BJ, LaRossa G, Sachs R, Fotenos AF, Sheline YI, Klunk WE, Mathis CA, Morris JC, Mintun MA (2005) Molecular, structural, and functional characterization of Alzheimer's disease: evidence for a relationship between default activity, amyloid, and memory. J Neurosci 25:7709-7717.

Busche MA, Eichhoff G, Adelsberger H, Abramowski D, Wiederhold KH, Haass C, Staufenbiel M, Konnerth A, Garaschuk O (2008) Clusters of hyperactive neurons near amyloid plaques in a mouse model of Alzheimer's disease. Science 321:1686-1689.

Celone KA, Calhoun VD, Dickerson BC, Atri A, Chua EF, Miller SL, DePeau K, Rentz DM, Selkoe DJ, Blacker D, Albert MS, Sperling RA (2006) Alterations in memory networks in mild cognitive impairment and Alzheimer's disease: an independent component analysis. J Neurosci 26:10222-10231.

Chételat G, Villemagne VL, Pike KE, Baron JC, Bourgeat P, Jones G, Faux NG, Ellis KA, Salvado O, Szoeke C, Martins RN, Ames D, Masters CL, Rowe CC (2010) Larger temporal volume in elderly with high versus low betaamyloid deposition. Brain 133:3349-3358.

Csernansky JG, Hamstra J, Wang L, McKeel D, Price JL, Gado M, Morris JC (2004) Correlations between antemortem hippocampal volume and postmortem neuropathology in AD subjects. Alzheimer Dis Assoc Disord 18:190-195.

Dale AM (1999) Optimal experimental design for event-related fMRI. Hum Brain Mapp 8:109-114.

de Chastelaine M, Wang TH, Minton B, Muftuler LT, Rugg MD (2011) The effects of age, memory performance, and callosal integrity on the neural correlates of successful associative encoding. Cereb Cortex 21:2166-2176.

Desikan RS, Sabuncu MR, Schmansky NJ, Reuter M, Cabral HJ, Hess CP, Weiner MW, Biffi A, Anderson CD, Rosand J, Salat DH, Kemper TL, Dale AM, Sperling RA, Fischl B (2010) Selective disruption of the cerebral neocortex in Alzheimer's disease. PLoS One 5:e12853.

Dickerson BC, Goncharova I, Sullivan MP, Forchetti C, Wilson RS, Bennett DA, Beckett LA, deToledo-Morrell L (2001) MRI-derived entorhinal and hippocampal atrophy in incipient and very mild Alzheimer's disease. Neurobiol Aging 22:747-754.

Dickerson BC, Salat D, Bates JF, Atiya M, Killiany R, Greve D, Dale AM, Stern CE, Blacker D, Albert M, Sperling R (2004) MRI measures of medial temporal lobe function and structure in questionable Alzheimer's disease. Ann Neurol 56:27-35.

Dickerson BC, Salat DH, Greve DN, Chua EF, Rand-Giovannetti E, Rentz DM, Bertram L, Mullin K, Tanzi RE, Blacker D, Albert MS, Sperling RA (2005) Increased hippocampal activation in mild cognitive impairment compared to normal aging and AD. Neurology 65:404-411.

Dickerson BC, Bakkour A, Salat DH, Feczko E, Pacheco J, Greve DN, Grodstein F, Wright CI, Blacker D, Rosas HD, Sperling RA, Atri A, Growdon JH, Hyman BT, Morris JC, Fischl B, Buckner RL (2009) The cortical signature of Alzheimer's disease: regionally specific cortical thinning relates to symptom severity in very mild to mild $\mathrm{AD}$ dementia and is detectable in asymptomatic amyloid-positive individuals. Cereb Cortex 19:497-510.

Dickerson BC, Stoub TR, Shah RC, Sperling RA, Killiany RJ, Albert MS, Hyman BT, Blacker D, Detoledo-Morrell L (2011) Alzheimer-signature MRI biomarker predicts AD dementia in cognitively normal adults. Neurology 76:1395-1402.

Drzezga A, Becker JA, Van Dijk KR, Sreenivasan A, Talukdar T, Sullivan C, Schultz AP, Sepulcre J, Putcha D, Greve D, Johnson KA, Sperling RA (2011) Neuronal dysfunction and disconnection of cortical hubs in nondemented subjects with elevated amyloid burden. Brain 134:1635-1646.

Du AT, Schuff N, Kramer JH, Rosen HJ, Gorno-Tempini ML, Rankin K, Miller BL, Weiner MW (2007) Different regional patterns of cortical thinning in Alzheimer's disease and frontotemporal dementia. Brain 130:1159-1166.

Duverne S, Motamedinia S, Rugg MD (2009) The relationship between aging, performance, and the neural correlates of successful memory encoding. Cereb Cortex 19:733-744.

Filippini N, MacIntosh BJ, Hough MG, Goodwin GM, Frisoni GB, Smith SM, Matthews PM, Beckmann CF, Mackay CE (2009) Distinct patterns of brain activity in young carriers of the APOE-epsilon4 allele. Proc Natl Acad Sci U S A 106:7209-7214.

Fischl B, Liu A, Dale AM (2001) Automated manifold surgery: constructing geometrically accurate and topologically correct models of the human cerebral cortex. IEEE Trans Med Imaging 20:70-80.

Fjell AM, Walhovd KB, Fennema-Notestine C, McEvoy LK, Hagler DJ, Holland D, Blennow K, Brewer JB, Dale AM (2010) Brain atrophy in healthy aging is related to CSF levels of Abeta1-42. Cereb Cortex 20:2069-2079.

Greicius MD, Supekar K, Menon V, Dougherty RF (2009) Resting-state functional connectivity reflects structural connectivity in the default mode network. Cereb Cortex 19:72-78.

Hämäläinen A, Pihlajamäki M, Tanila $\mathrm{H}$, Hänninen T, Niskanen E, Tervo S, Karjalainen PA, Vanninen RL, Soininen H (2007) Increased fMRI responses during encoding in mild cognitive impairment. Neurobiol Aging 28:1889-1903.

Hedden T, Van Dijk KR, Becker JA, Mehta A, Sperling RA, Johnson KA, Buckner RL (2009) Disruption of functional connectivity in clinically normal older adults harboring amyloid burden. J Neurosci 29:12686-12694

Holland D, Brewer JB, Hagler DJ, Fenema-Notestine C, Dale AM (2009) Subregional neuroanatomical change as a biomarker for Alzheimer's disease. Proc Natl Acad Sci U S A 106:20954-20959.

Johnson SC, Schmitz TW, Moritz CH, Meyerand ME, Rowley HA, Alexander AL, Hansen KW, Gleason CE, Carlsson CM, Ries ML, Asthana S, Chen K, Reiman EM, Alexander GE (2006) Activation of brain regions vulnerable to Alzheimer's disease: the effect of mild cognitive impairment. Neurobiol Aging 27:1604-1612.

Kahn I, Andrews-Hanna JR, Vincent JL, Snyder AZ, Buckner RL (2008) Distinct cortical anatomy linked to subregions of the medial temporal lobe revealed by intrinsic functional connectivity. J Neurophysiol 100:129-139.

Killiany RJ, Hyman BT, Gomez-Isla T, Moss MB, Kikinis R, Jolesz F, Tanzi R, Jones K, Albert MS (2002) MRI measures of entorhinal cortex vs hippocampus in preclinical AD. Neurology 58:1188-1196.

Kircher TT, Weis S, Freymann K, Erb M, Jessen F, Grodd W, Heun R, Leube DT (2007) Hippocampal activation in patients with mild cognitive impairment is necessary for successful memory encoding. J Neurol Neurosurg Psychiatry 78:812-818.

Klunk WE, Engler H, Nordberg A, Wang Y, Blomqvist G, Holt DP, Bergström M, Savitcheva I, Huang GF, Estrada S, Ausén B, Debnath ML, Barletta J, Price JC, Sandell J, Lopresti BJ, Wall A, Koivisto P, Antoni G, Mathis CA, Långström B (2004) Imaging brain amyloid in Alzheimer's disease with Pittsburgh Compound-B. Ann Neurol 55:306-319.

Kobayashi Y, Amaral DG (2003) Macaque monkey retrosplenial cortex: II. Cortical afferents. J Comp Neurol 466:48-79.

Logothetis NK, Wandell BA (2004) Interpreting the BOLD signal. Annu Rev Physiol 66:735-769.

Machulda MM, Ward HA, Borowski B, Gunter JL, Cha RH, O’Brien PC, Petersen RC, Boeve BF, Knopman D, Tang-Wai DF, Ivnik RJ, Smith GE, Tangalos EG, Jack CR Jr (2003) Comparison of memory fMRI response among normal, MCI, and Alzheimer's patients. Neurology 61:500-506.

Miller SL, Fenstermacher E, Bates J, Blacker D, Sperling RA, Dickerson BC (2008a) Hippocampal activation in adults with mild cognitive impairment predicts subsequent cognitive decline. J Neurol Neurosurg Psychiatry 79:630-635.

Miller SL, Celone K, DePeau K, Diamond E, Dickerson BC, Rentz D, Pihlajamäki M, Sperling RA (2008b) Age-related memory impairment associated with loss of parietal deactivation but preserved hippocampal activation. Proc Natl Acad Sci U S A 105:2181-2186.

Morcom AM, Good CD, Frackowiak RS, Rugg MD (2003) Age effects on the neural correlates of successful memory encoding. Brain 126:213-229.

Mormino EC, Smiljic A, Hayenga AO, Onami SH, Greicius MD, Rabinovici GD, Janabi M, Baker SL, Yen IV, Madison CM, Miller BL, Jagust WJ (2011) Relationships between beta-amyloid and functional connectivity in different components of the default mode network in aging. Cereb Cortex 21:2399-2407.

Morris JC (1993) The Clinical Dementia Rating (CDR): current version and scoring rules. Neurology 43:2412-2414.

Nestor PJ, Scheltens P, Hodges JR (2004) Advances in the early detection of Alzheimer's disease. Nat Med 10 [Suppl]:S34-S41.

O'Brien JL, O'Keefe KM, LaViolette PS, DeLuca AN, Blacker D, Dickerson BC, Sperling RA (2010) Longitudinal fMRI in elderly reveals loss of hippocampal activation with clinical decline. Neurology 74:1969-1976. 
Palop JJ, Mucke L (2010) Synaptic depression and aberrant excitatory network activity in Alzheimer's disease: two faces of the same coin? Neuromolecular Med 12:48-55.

Palop JJ, Chin J, Roberson ED, Wang J, Thwin MT, Bien-Ly N, Yoo J, Ho KO, Yu GQ, Kreitzer A, Finkbeiner S, Noebels JL, Mucke L (2007) Aberrant excitatory neuronal activity and compensatory remodeling of inhibitory hippocampal circuits in mouse models of Alzheimer's disease. Neuron 55:697-711.

Petrella JR, Krishnan S, Slavin MJ, Tran TT, Murty L, Doraiswamy PM (2006) Mild cognitive impairment: evaluation with 4-T functional MR imaging. Radiology 240:177-186.

Quiroz YT, Budson AE, Celone K, Ruiz A, Newmark R, Castrillón G, Lopera F, Stern CE (2010) Hippocampal hyperactivation in presymptomatic familial Alzheimer's disease. Ann Neurol 68:865-875.

Ries ML, Carlsson CM, Rowley HA, Sager MA, Gleason CE, Asthana S, Johnson SC (2008) Magnetic resonance imaging characterization of brain structure and function in mild cognitive impairment: a review. J Am Geriatr Soc 56:920-934.

Rossi S, Miniussi C, Pasqualetti P, Babiloni C, Rossini PM, Cappa SF (2004) Age-related functional changes of prefrontal cortex in long-term memory: a repetitive transcranial magnetic stimulation study. J Neurosci 24:7939-7944.

Sabuncu MR, Desikan RS, Sepulcre J, Yeo BT, Liu H, Schmansky NJ, Reuter M, Weiner MW, Buckner RL, Sperling RA, Fischl B (2011) The dynamics of cortical and hippocampal atrophy in Alzheimer disease. Arch Neurol 68:1040-1048.

Seeley WW, Crawford RK, Zhou J, Miller BL, Greicius MD (2009) Neurodegenerative diseases target large-scale human brain networks. Neuron 62:42-52.

Singh V, Chertkow H, Lerch JP, Evans AC, Dorr AE, Kabani NJ (2006) Spatial patterns of cortical thinning in mild cognitive impairment and Alzheimer's disease. Brain 129:2885-2893.
Sperling R, Chua E, Cocchiarella A, Rand-Giovannetti E, Poldrack R, Schacter DL, Albert M (2003) Putting names to faces: successful encoding of associative memories activates the anterior hippocampal formation. Neuroimage 20:1400-1410.

Sperling RA, Laviolette PS, O'Keefe K, O’Brien J, Rentz DM, Pihlajamaki M, Marshall G, Hyman BT, Selkoe DJ, Hedden T, Buckner RL, Becker JA, Johnson KA (2009) Amyloid deposition is associated with impaired default network function in older persons without dementia. Neuron 63:178-188.

Sperling RA, Dickerson BC, Pihlajamaki M, Vannini P, LaViolette PS, Vitolo OV, Hedden T, Becker JA, Rentz DM, Selkoe DJ, Johnson KA (2010) Functional alterations in memory networks in early Alzheimer's disease. Neuromolecular Med 12:27-43.

Sperling RA, Aisen PS, Beckett LA, Bennett DA, Craft S, Fagan AM, Iwatsubo T, Jack CR Jr, Kaye J, Montine TJ, Park DC, Reiman EM, Rowe CC, Siemers E, Stern Y, Yaffe K, Carrillo MC, Thies B, Morrison-Bogorad M, Wagster MV, Phelps CH (2011) Toward defining the preclinical stages of Alzheimer's disease: recommendations from the national institute on aging and the Alzheimer's association workgroup. Alzheimers Dement 7:280-292.

Vannini P, Hedden T, Becker JA, Sullivan C, Putcha D, Rentz D, Johnson KA, Sperling RA (2011) Age and amyloid-related alterations in default network habituation to stimulus repetition. Neurobiol Aging. Advance online publication. doi:10.1016/j.neurobiolaging.2011.01.003.

Vincent JL, Snyder AZ, Fox MD, Shannon BJ, Andrews JR, Raichle ME, Buckner RL (2006) Coherent spontaneous activity identifies a hippocampal-parietal memory network. J Neurophysiol 96:3517-3531.

Yassa MA, Stark SM, Bakker A, Albert MS, Gallagher M, Stark CE (2010) High-resolution structural and functional MRI of hippocampal CA3 and dentate gyrus in patients with amnestic mild cognitive impairment. Neuroimage 51:1242-1252. 